\title{
Peer Reviewers
}

The following is the list of people who served as peer reviewers during 2009 (Vol. 63: 1-4). The number in parentheses indicates those who graciously agreed to review more than one manuscript. I apologize for anyone who has been left off. Reviewing research articles is a time consuming and usually thankless task. By publishing your names, the editors and staff of Economic Botany say—-thanks!

\section{Raf Aerts}

Ulysses Albuquerque (3)

Vilela Alejandra

Miguel Alexiades

Wendy Applequist (2)

Daniel F. Austin (2)

William Balée

Michael Balick

AP Belchior

Bradley Bennett

Bruce Benz

Brent Berlin

Stephen Brush

Alejandro Casas

Charles R. Clement

Michelle Cocks

Felix Coe

Oliver Coomes

Gary Crawford

Anthony Cunningham (3)

Marie Christine Daunay

George Estabrook (2)

Barbara Frei

Orou Gaoue

Martha Sofía González-Insuasti

Roy Hamilton

Bryan A. Hanson

Neil Harriman

Donald L. Hazlett
Michael Heinrich
Elisabeth Hildebrand
Wendy Hodgson
Verina Ingram
Barrie Juniper
Eric Kightley
Sabrina Krief
Ana Haydeé Ladio
Wayne Law
David Lentz
Dana Lepofsky
Pesach Lubinsky(2)
Lisa Mandle
Eric Okuku Manyasa
Steven McKean
Heather McKillop
Rebecca McLain
Will McClatchey
Heather McMillen
William Milliken
Dan Moerman
Jocelyn Grupp Mueller
Lee Newsome
My Lien Nguyen
Manuel Pardo
Kathy Parker
David Pilz

Ghillean T. Prance

Maria Teresa Pulido

John Rashford (2)

Dave Reedy

Karl Rensch

Victoria Reyes-Garcia

María de los Reyes

Gonzáles-Tejero

Robert Rhoades

Nanci Ross

Joy Runyon

Sy Sohmer

Rick Stepp

Kristine Stewart

John Robert Stephen Tabuti

Michael Thomas

Tamara Ticktin

Patrick van Damme

Ina Vandebroek

Julie Velasquez-Runk

Christian Reinhard Vogl

Gail Wagner

Yoav Waisel

Brad Walters

Caroline S. Weckerle

Alexander Wezel

Wayne Arthur Whistler

Scott Zona 